\title{
Conditional Volatility Targeting
}

\section{Dion Bongaerts, Xiaowei Kang \& Mathijs van Dijk}

To cite this article: Dion Bongaerts, Xiaowei Kang \& Mathijs van Dijk (2020): Conditional Volatility Targeting, Financial Analysts Journal, DOI: 10.1080/0015198X.2020.1790853

To link to this article: https://doi.org/10.1080/0015198X.2020.1790853

$$
\begin{aligned}
& \text { (c) } 2020 \text { The Author(s). Published with } \\
& \text { license by Taylor \& Francis Group, LLC. }
\end{aligned}
$$

\section{$+$}

View supplementary material $\sqsubset$

曲 Published online: 04 Sep 2020.

Submit your article to this journal $\pi$

Џlll Article views: 702

Q View related articles $\asymp$

View Crossmark data $\nearrow$ 


\section{Conditional Volatility Targeting}

\section{Dion Bongaerts $₫$, Xiaowei Kang, CFA, and Mathijs van Dijk $₫$}

Dion Bongaerts is an associate professor of finance at Rotterdam School of Management, Erasmus University, the Netherlands. Xiaowei Kang, CFA, is head of research of the Indexed Funds Department at the Abu Dhabi Investment Authority and an affiliated researcher at Rotterdam School of Management, Erasmus University, the Netherlands. Mathijs van Dijk is a professor of finance at Rotterdam School of Management, Erasmus University, the Netherlands.

In analyzing the performance of volatility-targeting strategies, we found that conventional volatility targeting fails to consistently improve performance in global equity markets and can lead to markedly greater drawdowns. Motivated by return patterns in various volatility states, we propose a strategy of conditional volatility targeting that adjusts risk exposures only in the extremes during high- and low-volatility states. This strategy consistently enhances Sharpe ratios and reduces drawdowns and tail risks, with low turnover and leverage, when used in the major equity markets and for momentum factors across regions. Conditional volatility management can also be applied to tactical allocations among multiple assets or risk factors.

This is an Open Access article distributed under the terms of the Creative Commons Attribution-NonCommercial-NoDerivatives License (http://creativecommons.org/licenses/ by-nc-nd/4.0/), which permits non-commercial re-use, distribution, and reproduction in any medium, provided the original work is properly cited, and is not altered, transformed, or built upon in any way.

Disclosure: The authors report no conflicts of interest. The views expressed in this article are those of the authors in their personal capacity and do not reflect the views of the Rotterdam School of Management or the Abu Dhabi Investment Authority.
T he clustering of volatility and the negative correlation between realized volatility and returns are two well-documented features of equity markets. These features have motivated the use of volatility targeting to dynamically adjust market exposure based on volatility. Volatility targeting essentially levers up the exposure whenever volatility is low and scales it down when volatility is high. Investors can use this strategy to boost portfolio returns and mitigate tail risks and drawdowns.

Against the recent backdrop of elevated macroeconomic and market uncertainties, volatility-targeting strategies have received renewed attention among both academics and practitioners. Moreira and Muir (2017) found that volatility-managed portfolios increase Sharpe ratios for the US equity market and long-short equity factors. Harvey, Hoyle, Korgaonkar, Rattray, Sargaison, and Van Hemert (2018) showed that volatility targeting reduces tail risks across asset classes in the US market but that the Sharpe ratio gain is limited to "risk assets" (equities and corporate credit).

In our study, we critically assessed the performance and practical implementation of volatility-targeting strategies in major equity markets and for major factors. Our strategies use equity index futures and investable equity factors so that they can be implemented in practice. In this article, we first confirm the findings of Liu, Tang, and Zhou (2019) that the volatility-targeting strategies in Moreira and Muir (2017) and Harvey et al. (2018) are subject to look-ahead bias because they involve a scaling factor that is constructed ex post. We describe an implementable volatility-targeting strategy we constructed that does not involve ex post scaling, which we call conventional volatility targeting. We found that the conventional volatility-targeting strategy does not consistently improve risk-adjusted performance in international equity markets and can significantly overshoot the volatility target-thereby increasing maximum drawdowns and tail risks. When applying the conventional strategy to equity factors, we found that it increased Sharpe ratios for the momentum factor (factors are defined later) across US 
and international markets, but it did not do so for the size, value, profitability, or investment factors. Furthermore, the conventional strategy is associated with high portfolio turnover (often more than 200\% per year) and significant time-varying leverage, which is likely to cause problems in practical implementation. These findings cast doubt on the usefulness of conventional volatility-targeting strategies.

We continue in the article by showing that the gains from volatility targeting vary with volatility states. In particular, during times of high volatility, volatility clustering is stronger, the correlation between realized volatility and future returns is significantly more negative, and the gains from volatility targeting are thus larger than in times of low or medium volatility. Motivated by these findings, we propose a conditional volatility-targeting strategy that adjusts risk exposures conditioned on (extreme) volatility states. In its basic form, the strategy reduces risk exposures during high-volatility states, increases risk exposures during low-volatility states, and maintains an unscaled exposure otherwise.

We show that this conditional strategy significantly reduces drawdowns and tail risks in all major equity markets and for the momentum factor, and it does so with significantly lower turnover and leverage than the conventional volatility-targeting strategy. Furthermore, we show that the conditional strategy enhances Sharpe ratios-most prominently for the momentum factor across regions and, to a lesser degree, for the market factor. We also show that the performance of the conditional strategy is robust to alternative approaches to identifying volatility states.
We conclude that conditioning on (extreme) volatility states is important when using volatility targeting. Importantly, investors should be selective with the use of volatility targeting because the strategy improves performance only for a limited range of factors and markets.

Conditional volatility management has broader applications than discussed in our main analysis. Volatility management that allocates between risky assets (equities, momentum-factor strategies) and flight-toquality assets (treasuries, profitability-factor strategies) during high-volatility states can enhance returns and is a promising direction for future research.

\section{Data and Methods}

We used daily return data on global equity markets and factors. Exhibit 1 provides an overview of our data. For equity markets, we covered the 10 largest markets that historically account for more than $80 \%-90 \%$ of market capitalization in global equities. ${ }^{1}$ These markets have liquid equity index futures, which significantly reduces the cost of implementing volatility-targeting strategies. Our daily returns are given in domestic currencies and cover the period of January 1972 to March 2019. We used equity index futures after their inception in each market; prior to that time, we used the corresponding equity index returns for the market. $^{2}$

For equity factors, we used data from Kenneth French's website to construct (large-capitalization versions of) the following factors: momentum (MOM, or Carhart 1997 monthly momentum), size

\section{Exhibit 1. Sample and Data Description}

\begin{tabular}{|c|c|c|c|c|}
\hline Assets & Regions & $\begin{array}{c}\text { Complete } \\
\text { Sample Period }\end{array}$ & $\begin{array}{c}\text { Strategy } \\
\text { Sample Period }\end{array}$ & Data Source \\
\hline $\begin{array}{l}\text { Equity markets } \\
\text { (equity index } \\
\text { futures) }\end{array}$ & $\begin{array}{l}\text { US, Japan, UK, France, Canada, } \\
\text { Germany, Switzerland, } \\
\text { Australia, Netherlands, } \\
\text { and Hong Kong SAR }\end{array}$ & 1972-2019 & 1982-2019 & Datastream \\
\hline \multirow{2}{*}{$\begin{array}{l}\text { Equity factors (size, } \\
\text { value, profitabil- } \\
\text { ity, investment, } \\
\text { and momentum) }\end{array}$} & US & 1963-2019 & 1973-2019 & \multirow[b]{2}{*}{$\begin{array}{l}\text { Kenneth R. French website } \\
\text { (https://mba.tuck.dart- } \\
\text { mouth.edu/pages/faculty/ } \\
\text { ken.french/data_library. } \\
\text { html); factor returns recon- } \\
\text { structed by authors using } \\
\text { only the "Big" portfolios to } \\
\text { ensure investability }\end{array}$} \\
\hline & $\begin{array}{l}\text { Global, Global ex US, Europe, } \\
\text { Japan, Asia Pacific ex Japan }\end{array}$ & 1990-2019 & 1995-2019 & \\
\hline
\end{tabular}


(SMB, or small minus big), value (HML, or high book to market minus low book to market), profitability (RMW, or robust [high] operating profitability minus weak [low] operating profitability), and investment (CMA, or conservative investment minus aggressive investment); see Fama and French (2015). ${ }^{3}$ The data (based on availability of return data) span July 1963-March 2019 for the US factors and November 1990-March 2019 for global, global ex US, Europe, Japan, and Asia ex Japan factors.

The factors used in most academic studies are well known to be not investable strategies because they include many small- and microcap stocks. Such stocks are generally illiquid and difficult to short. Therefore, for all the equity factors except SMB, we recalculated factor returns using the largest stocks with respect to market capitalization (largerthan-median stocks by market cap on the NYSE). For instance, whereas the original $\mathrm{HML}$ factor is constructed as $[1 / 2 \times($ Small Value + Big Value $)]-[1 / 2 \times$ (Small Growth + Big Growth), we reconstructed the factor as Big Value - Big Growth. For the size factor, we formed portfolios on the basis of market cap and constructed the factor as the difference between the largest size quintile and the second-largest quintile. ${ }^{4}$

Following Moreira and Muir (2017) and Harvey et al. (2018), we focused on excess returns. Returns of equity index futures and long-short equity factors are equivalent to excess returns because both are unfunded. To calculate excess returns, we used for the period prior to the inception of equity index futures the cash equity index returns and deducted the risk-free rate (the three-month T-bill rate for the US market and the equivalent for other countries/ regions, all of which came from Datastream).

\section{Constructing Volatility-Targeting}

Portfolios. Volatility-targeting strategies scale the excess returns of the original portfolio by the inverse of its conditional volatility. Consistent with the findings of Liu et al. (2019), however, we point out that the strategies constructed by Moreira and Muir (2017) and Harvey et al. (2018) are subject to lookahead bias as to volatility because their methodology assumes that the volatility-targeting portfolio can be rescaled ex post. For instance, Harvey et al. (2018) calculated volatility-scaled returns as follows:

$$
r_{t}^{\text {scaled }}=r_{t} \times \frac{\sigma^{\text {target }}}{\sigma_{t-2}} \times k^{\text {scaled }}
$$

where $r_{t}$ is return at time $t, \sigma$ is standard deviation, $\sigma^{\text {target }}$ is a fixed volatility target, and the constraint $k^{\text {scaled }}$ is chosen ex post to achieve the specific volatility target over the full sample period. Under certain assumptions, ${ }^{5}$ ex post rescaling leaves Sharpe ratios unaffected and the performance of the strategy easy to compare with the original portfolio, but the strategies are impossible to implement in practice.

Moreover, they may leave investors exposed to unanticipatedly high volatility and excess kurtosis of returns. Therefore, we constructed implementable volatility-targeting strategies without ex post scaling and look-ahead bias.

Specifically, we constructed two types of volatilitytargeting strategies. The first strategy (which we call "conventional volatility targeting" and for which we, hereafter, use the shorthand "conventional strategy") scales risk exposure throughout the sample period at the start of each month $t$ :

$$
r_{t}^{\text {scaled }}=r_{t} \times \frac{\sigma^{\text {target }}}{\hat{\sigma}_{t-1}} \text { for } t=1 \text { to } T
$$

where $\sigma^{\text {target }}$ is the long-term realized volatility calculated using all daily returns up to and including month $t-1$. The variable $\hat{\sigma}_{t-1}$ is the predicted volatility for month $t$ and was estimated by the realized volatility in the previous month, $t-1$ (as the equally weighted standard deviation of daily returns). We excluded the last trading day of the month to ensure that $\hat{\sigma}_{t-1}$ would be known one day ahead of rebalancing.

The second strategy ("conditional volatility targeting," or the "conditional strategy") adjusts risk exposures only when month $t$ is predicted to be an extreme volatility state (scaling up to a certain leverage), and otherwise, an unscaled risk exposure is maintained; that is,

$$
r_{t}^{\text {scaled }}=I_{t} \times r_{t} \times \min \left(\frac{\sigma^{\text {target }}}{\hat{\sigma}_{t-1}}, L_{\max }\right)+\left(1-I_{t}\right) \times r_{t},
$$

where indicator variable $I_{t}$ equals 1 if month $t$ is predicted to be an extreme volatility state and equals 0 otherwise and $L_{\max }$ is the maximum leverage the strategy can take on. We constructed the conditional strategy to reduce exposure and risk in high-volatility states, increase exposure and risks by taking on leverage in low-volatility states, and maintain an unscaled exposure in medium-volatility states. ${ }^{6}$ 
To forecast volatility states for the conditional strategy, we sorted realized volatility of all months up to $t-1$ into quintiles. If the volatility of month $t-1$ was in the highest (lowest) quintile, we predicted a highvolatility (low-volatility) state for month $t$; otherwise, we predicted a medium-volatility state for month $t$. This simple approach used the same data as the conventional strategy, so the strategies are directly comparable. In addition, our conditional strategy is robust to the use of alternative bands for predicted volatility states-such as realized volatility quartiles instead of quintiles-or fixed ratios of realized volatility over target volatility (see the section "Robustness Tests of Conditional Strategies").

For analysis of both equity markets and equity factors, we set the volatility target in month $t$ as, based on all return data available prior to month $t$, the longterm volatility of that asset. This approach prevented any look-ahead bias or arbitrary choices as to volatility targets. We required at least 10 years of returns for calculating the long-term volatility. Therefore, our volatility-targeting analysis starts 10 years after the starting dates of the two datasets. ${ }^{7}$

As a robustness check, we also followed Harvey et al. (2018) by using the exponentially weighted standard deviation of daily returns in the previous year to estimate volatility. In addition, we used GARCH (generalized autoregressive conditional heteroskedasticity) forecasts to estimate volatility. We found the benefits of volatility targeting to be robust to different volatility forecasts. We preferred using realized volatility in the previous month for simplicity reasons. ${ }^{8}$ Implied volatility is a potentially more efficient estimate of future volatility that may further improve performance but is not used in this article because it is available only for a short sample period for the 10 equity markets we studied; it is not available for the long-short equity factors.

\section{Transaction Costs. All of our performance} measures are net of transaction costs. To account for the trading costs for implementing the volatilitytargeting strategies, we assumed a transaction cost of 3 basis points (bps) of notional value traded for equity futures for the last 20 years of the sample period and 5 bps for the earlier period, which is more conservative than the 1 bp used by Harvey et al. (2018). We based our assumption on Kotecha, Couasnon, and Galazidis (2016) and CME Group (2016), which estimated a total transaction cost of 1.4-1.5 bps for a $\$ 100$ million order on S\&P 500 Index futures (these estimates included market impact). Trading costs in international equity index futures are probably higher than in US markets, but the market impact may be mitigated because of smaller transaction sizes.

For trading equity factors, the transaction costs contain two components. The first comes from the trading associated with volatility targeting. The second comes from the rebalancing of the equity factors themselves. Neither the Fama-French factor returns on the French website nor Moreira and Muir (2017) account for the latter. For the first component, we assumed a transaction cost of 25 bps of notional value traded to implement volatility targeting, which is more conservative than the 18 bps average implementation shortfall found by Frazzini, Israel, and Moskowitz (2015). ${ }^{9}$ We used a more conservative estimate to account for higher trading costs in the earlier periods of our sample. For the second component, we conservatively assumed an annualized two-way turnover of $400 \%$ for momentum (which translates to 100 bps of rebalancing cost) and $200 \%$ for other factors (translating to 50 bps of rebalancing cost).

\section{Motivation for Conditional Volatility Targeting}

In this section, we review the performance drivers of volatility targeting, consider the challenges of implementing the conventional strategy, and discuss motivation for the conditional strategy.

\section{Conventional Volatility Targeting:} Inconsistent Performance in Equity Markets. Two well-documented features of equity markets underpin the theory and practice of volatility targeting. The first is volatility clustering, which led to the development of autoregressive conditional heteroskedasticity models (Engle 1982), which are now widely used in financial economics. The second is the negative correlation between volatility and returns. In particular, market crashes tend to be associated with volatility spikes. ${ }^{10}$ Black (1976) explained the negative contemporaneous correlation between equity returns and volatility by noting a "leverage effect"; that is, negative returns increase financial leverage, leading to increased risk and volatility. ${ }^{11}$

Harvey et al. (2018) argued that the leverage effect is confined to risk assets (stocks and corporate bonds) and can explain why volatility targeting improves Sharpe ratios for such assets. Yet, the contemporaneous correlation between returns and volatility 
cannot be directly exploited. Instead, volatilitytargeting strategies use estimates of future volatility, such as realized volatility. Therefore, it is the correlation between realized volatility and future returns (as well as future realized volatility) that largely determines whether volatility targeting leads to superior risk-adjusted returns. ${ }^{12}$

An analysis of the performance of conventional volatility targeting provides insights into these two performance drivers. Table 1 shows performance statistics for the conventional volatility-targeting strategy versus the original portfolio in major equity markets and for US equity factors. In addition to the Sharpe ratio, we report the maximum drawdown and the expected shortfall (99\%) because one of the main motivations of the strategy is to reduce downside risk and tail risk..$^{13}$ For ease of comparison, we show the performance of the conventional strategy relative to the original portfolios. We highlighted in boldface any performance results shown in Table 1 that contradict the objectives of the strategy. Also reported is the ratio of realized volatility to target volatility for the volatility-targeting strategies; the unconditional volatility estimate of the original portfolio is the target.

Panel A of Table 1 shows that the conventional strategy improves Sharpe ratios the most in the US and Japanese markets; the improvement is much weaker or even nonexistent in other markets.

\section{Table 1. Performance of the Conventional Volatility-Targeting Strategy}

Original Portfolio

\begin{tabular}{|c|c|c|c|c|c|c|c|}
\hline $\begin{array}{c}\text { Sharpe } \\
\text { Ratio }\end{array}$ & $\begin{array}{l}\text { Maximum } \\
\text { Drawdown }\end{array}$ & $\begin{array}{l}\text { Expected } \\
\text { Shortfall }\end{array}$ & $\begin{array}{c}\text { Sharpe } \\
\text { Ratio } \\
\text { Increase }\end{array}$ & $\begin{array}{l}\text { Maximum } \\
\text { Drawdown } \\
\text { Reduction }\end{array}$ & $\begin{array}{c}\text { Expected } \\
\text { Shortfall } \\
\text { Reduction }\end{array}$ & $\begin{array}{l}\text { Realized } \\
\text { Volatility/ } \\
\text { Target }\end{array}$ & $\begin{array}{l}\text { Annual } \\
\text { Turnover } \\
\text { (times) }\end{array}$ \\
\hline
\end{tabular}

A. Global equity markets (1982-2019)

\begin{tabular}{|c|c|c|c|c|c|c|c|c|}
\hline US & 0.59 & $52.8 \%$ & $-14.9 \%$ & $0.15^{*}$ & $-7.0 \%$ & $1.2 \%$ & 1.16 & 2.4 \\
\hline Japan & 0.10 & 75.4 & -17.1 & 0.13 & -2.8 & -1.8 & 0.98 & 1.8 \\
\hline UK & 0.43 & 49.4 & -14.9 & 0.01 & 9.8 & 1.5 & 1.31 & 2.1 \\
\hline France & 0.38 & 60.7 & -16.6 & 0.00 & -3.6 & 1.3 & 1.05 & 1.9 \\
\hline Canada & 0.35 & 49.6 & -17.2 & 0.01 & 4.0 & 1.9 & 1.15 & 2.1 \\
\hline Germany & 0.36 & 68.3 & -20.4 & 0.06 & -9.9 & 0.8 & 0.99 & 1.8 \\
\hline Switzerland & 0.42 & 51.3 & -16.6 & 0.06 & -0.8 & 0.2 & 1.09 & 2.2 \\
\hline Australia & 0.42 & 51.0 & -18.4 & -0.03 & 4.0 & 6.4 & 1.34 & 2.1 \\
\hline Netherlands & 0.36 & 68.7 & -21.2 & 0.01 & -4.8 & -0.3 & 1.14 & 2.3 \\
\hline Hong Kong SAR & 0.31 & 59.4 & -28.3 & -0.04 & 34.4 & 16.9 & 1.57 & 2.6 \\
\hline Market average & 0.37 & 58.7 & -18.6 & 0.04 & 2.3 & 2.8 & 1.18 & 2.1 \\
\hline \multicolumn{9}{|c|}{ B. US equity factors (1973-2019) } \\
\hline Size & 0.25 & $46.5 \%$ & $-5.9 \%$ & $-0.15^{* *}$ & $3.8 \%$ & $0.0 \%$ & 0.99 & 1.6 \\
\hline Value & 0.13 & 42.9 & -10.2 & $-0.13^{*}$ & 19.2 & -0.4 & 0.95 & 1.7 \\
\hline Profitability & 0.19 & 34.6 & -7.2 & -0.08 & 1.0 & -0.3 & 0.95 & 1.6 \\
\hline Investment & 0.26 & 48.5 & -9.2 & $-0.22^{* *}$ & 17.5 & -1.7 & 1.00 & 2.0 \\
\hline Momentum & 0.14 & 51.3 & -18.0 & $0.16^{* *}$ & -7.4 & -5.5 & 0.93 & 2.0 \\
\hline
\end{tabular}

Notes: Results are net of transaction costs. The "Expected Shortfall" (99\%) is the mean of monthly returns below the first percentile. "Realized Volatility/Target" is the ratio of the realized volatility of the strategy to the volatility of the original portfolio. "Annual Turnover" is one-way turnover (i.e., the sum of buy and sell divided by two). Performance results that violated the objectives of the strategy are in boldface. For statistics on Sharpe ratios, see Lo (2002).

${ }^{*}$ Sharpe ratio increase significant at the $5 \%$ level.

${ }^{* *}$ Sharpe ratio increase significant at the $1 \%$ level. 
The conventional strategy reduces maximum drawdown the most in the US and German markets, and it actually increases the maximum drawdown in the UK, Canadian, Australian, and Hong Kong markets, by $4.0 \%-34.4 \%$. The strategy also increases expected shortfall in 8 out of 10 markets, including the US market. This result defies one of the main purposes of volatility targeting: reduction of downside risk. This result is at odds with Harvey et al. (2018), who showed that volatility targeting consistently reduces tail risk. A possible reason is their ex post scaling.

Volatility-targeting strategies can over- or undershoot the target volatility in the following month. The ratio of realized volatility to target volatility of the conventional strategy in Panel A of Table 1 is generally higher than 1.0 (e.g., 1.16 in the US market and 1.31 in the UK market), which suggests that overshooting has more significant effects on the realized volatility of the conventional strategy than undershooting. ${ }^{14}$ Overshooting target volatility can cause problems in practice. For instance, it can completely distort the risk contribution of equities in an investor's overall asset allocation. In addition, it is the main driver of the increased drawdown and/or tail risk we observe.

The average annual turnover of the conventional strategy shown in Panel A of Table 1 is high-ranging from 1.8 to 2.6 in various markets.

Overall, we conclude that there is no evidence that the conventional volatility-targeting strategy consistently increases risk-adjusted returns and reduces downside risk across international markets. The lack of consistent outperformance and risk reduction, together with the high turnover of the conventional strategy, makes conventional volatility targeting unappealing for implementation.

Panel B of Table 1 shows results for our analysis of the performance of volatility-targeting strategies for equity factors in the US market. Since the report by Ang, Goetzmann, and Schaefer (2009), factor investing has become increasingly popular among institutional investors. Because equity factors are cyclical and can experience large drawdowns, investors typically diversify among multiple factors to reduce downside risk. Another approach to managing the downside risk of factor portfolios is to dynamically adjust the exposure to individual factors on the basis of the state of the market. Volatility management is a natural candidate for achieving such downside risk reduction.
Panel B of Table 1 shows the performance of the conventional volatility-targeting strategy versus the original long-short factor portfolios. For the US momentum factor, the strategy significantly improved the Sharpe ratio (by 0.16), decreased the maximum drawdown by $7.4 \%$ (from $51.3 \%$ ), and reduced the expected shortfall by $5.5 \%$ (from $-18.0 \%) .{ }^{15}$ For the US size, value, and investment factors, however, conventional volatility targeting decreased the Sharpe ratios and increased the maximum drawdowns.

We conducted a similar analysis for equity factors in international markets. Consistent with our findings for the US factors and with a concurrent paper by Cederburg, O'Doherty, Wang, and Yan (forthcoming), the conventional strategy improved Sharpe ratios and reduced downside risk for the momentum factor in various regions but did not do so for other factors. ${ }^{16}$ This finding is in contrast to Moreira and Muir (2017), who found that volatility-managed portfolios produce large alphas for all equity factors. Importantly, the conventional strategy requires significant and time-varying leverage and incurs high turnover. For the momentum factor, the maximum leverage ranges between 3.7 and 5.5, and the annualized turnover ranges between 2.0 and 2.6. These characteristics make the conventional strategy particularly challenging to implement for long-short equity factors because of trading and shorting costs.

\section{The Behavior of Asset Prices Differs} across Volatility States. The lack of performance consistency in the conventional strategies can be attributed to the relationship between volatility and return. Table 2 provides data on the behavior of returns and volatility in the three different volatility states. Panel A of Table 2 shows that the (tradable) correlation between realized volatility and future returns is only -0.02 , on average, in the major equity markets (compared with a strongly negative contemporaneous correlation of approximately -0.30 ). This relationship explains the relatively weak and inconsistent performance of conventional strategies in global markets.

Panels $B$ and $C$ of Table 2 show that the correlations between volatility and future returns are strongly negative for the momentum factor across regions but not for any other factors. To further illustrate this phenomenon, Figure A3 in Appendix A shows that momentum crashes are often associated with spikes in momentum volatility-most notably in 2009 but also in other episodes, such as 2000-2001. 


\section{Table 2. Behavior of Returns and Volatilities in Different Volatility States}

\begin{tabular}{|c|c|c|c|c|c|c|c|c|}
\hline & \multirow{2}{*}{$\begin{array}{c}\text { Contemporaneous } \\
\text { Correlation } \\
\text { (volatility vs. } \\
\text { return) }\end{array}$} & \multicolumn{4}{|c|}{$\begin{array}{c}\text { Correlation of Realized Volatility } \\
\text { with Future Return }\end{array}$} & \multicolumn{3}{|c|}{ Volatility Autocorrelation } \\
\hline & & $\begin{array}{l}\text { Whole } \\
\text { Period }\end{array}$ & $\begin{array}{l}\text { High- } \\
\text { Vol. } \\
\text { States }\end{array}$ & $\begin{array}{l}\text { Medium- } \\
\text { Vol. } \\
\text { States }\end{array}$ & $\begin{array}{l}\text { Low- } \\
\text { Vol. } \\
\text { States }\end{array}$ & $\begin{array}{l}\text { High- } \\
\text { Vol. } \\
\text { States }\end{array}$ & $\begin{array}{l}\text { Medium- } \\
\text { Vol. } \\
\text { States }\end{array}$ & $\begin{array}{l}\text { Low- } \\
\text { Vol. } \\
\text { States }\end{array}$ \\
\hline \multicolumn{9}{|c|}{ A. Global equity markets } \\
\hline US & -0.32 & -0.05 & -0.22 & -0.02 & -0.06 & 0.43 & 0.14 & 0.22 \\
\hline Japan & -0.33 & -0.06 & -0.04 & 0.11 & -0.02 & 0.35 & 0.04 & 0.22 \\
\hline UK & -0.14 & 0.04 & -0.05 & -0.01 & 0.01 & 0.60 & 0.13 & 0.13 \\
\hline France & -0.30 & -0.01 & -0.17 & 0.02 & 0.08 & 0.52 & 0.18 & 0.24 \\
\hline Canada & -0.32 & -0.05 & -0.18 & 0.00 & -0.05 & 0.66 & 0.15 & 0.31 \\
\hline Germany & -0.30 & 0.02 & -0.14 & -0.02 & -0.07 & 0.55 & 0.20 & 0.26 \\
\hline Switzerland & -0.35 & -0.03 & -0.10 & -0.01 & 0.09 & 0.45 & 0.05 & 0.31 \\
\hline Australia & -0.31 & -0.03 & -0.08 & 0.03 & -0.14 & 0.59 & 0.07 & 0.26 \\
\hline Netherlands & -0.37 & -0.01 & -0.09 & -0.10 & 0.07 & 0.66 & 0.13 & 0.15 \\
\hline Hong Kong SAR & -0.23 & -0.03 & 0.02 & -0.03 & -0.04 & 0.35 & 0.35 & 0.21 \\
\hline Market average & -0.30 & -0.02 & -0.10 & 0.00 & -0.01 & 0.52 & 0.14 & 0.23 \\
\hline \multicolumn{9}{|l|}{ B. US equity factors } \\
\hline Size & -0.12 & 0.06 & 0.05 & 0.01 & 0.03 & 0.42 & 0.12 & 0.26 \\
\hline Value & 0.07 & 0.02 & 0.05 & 0.01 & -0.17 & 0.81 & 0.13 & 0.19 \\
\hline Profitability & 0.14 & 0.09 & 0.21 & -0.03 & 0.01 & 0.69 & 0.14 & 0.08 \\
\hline Investment & 0.19 & 0.17 & 0.22 & 0.02 & -0.01 & 0.71 & 0.08 & 0.12 \\
\hline Momentum & -0.23 & -0.14 & -0.21 & -0.06 & 0.16 & 0.71 & 0.18 & 0.23 \\
\hline \multicolumn{9}{|c|}{ C. Global momentum factors } \\
\hline Global & -0.30 & -0.16 & -0.21 & 0.06 & 0.10 & 0.65 & 0.08 & 0.29 \\
\hline Global ex US & -0.24 & -0.15 & -0.22 & -0.02 & 0.07 & 0.62 & 0.22 & 0.22 \\
\hline Europe & -0.20 & -0.19 & -0.29 & -0.07 & -0.04 & 0.70 & 0.11 & 0.24 \\
\hline Japan & -0.18 & -0.05 & -0.06 & 0.09 & 0.09 & 0.52 & 0.13 & 0.07 \\
\hline Pacific ex Japan & -0.31 & -0.25 & -0.27 & 0.07 & -0.19 & 0.37 & 0.15 & 0.23 \\
\hline $\begin{array}{l}\text { Momentum } \\
\text { average }\end{array}$ & -0.24 & -0.16 & -0.21 & 0.01 & 0.03 & 0.60 & 0.15 & 0.21 \\
\hline
\end{tabular}

Notes: The realized volatility of month $t-1$ was sorted into five quintiles as proxies for different volatility states. The lowestand highest-volatility quintiles are defined as, respectively, the low-volatility and high-volatility states. To save space, statistics reported for the medium-volatility state are the average of Quintiles 2-4. "Volatility Autocorrelation" is the correlation between the realized volatility of current and previous months.

In comparison, the profitability factor tends to do well when volatility is high; it generated significant returns during both periods.

As discussed, the other practical problems of conventional volatility targeting include the tendency to overshoot the volatility target, increased drawdowns and tail risk, significant leverage, and high turnover. We argue that these problems are directly related to the constant and unconditional rebalancing across all volatility states. Such unconditional rebalancing implicitly assumes a uniform utility gain from volatility targeting across all volatility states. 
In contrast, it is well documented that economic and volatility states significantly affect investor behavior and asset prices. For instance, Henkel, Martin, and Nardari (2011) showed that short-horizon return predictability is weak in good times but sizable in bad times, when volatility tends to be high. Chen and Liang (2007) examined market timing by hedge funds and found that their timing ability appears stronger in bear and volatile market conditions. Ang and Bekaert (2004) and Kritzman, Page, and Turkington (2012) related shifts in volatility states and economic regimes to the associated opportunities for dynamic asset allocation.

Motivated by these findings, we examined the behavior of returns and volatilities in different volatility states. We report in Table 2 the tradable correlation between realized volatility and future returns for different volatility states as defined in the section "Constructing Volatility-Targeting Portfolios." Panel A shows that this correlation is more negative in high-volatility states than low-volatility states across equity markets. For instance, for the US market, it is -0.22 in the high-volatility state and -0.02 and -0.06 in, respectively, the medium-volatility and low-volatility states. Averaged across markets, this correlation equals -0.10 in the high-volatility state, compared with 0.00 and -0.01 , respectively, in the medium- and low-volatility states. Panels B and C of Table 2 report these correlations for equity factors. We focused on the momentum factor and found that the correlation between realized volatility and future returns is much more negative in high-volatility states across regions (on average, -0.21 , compared with an average of 0.01 and 0.03 in medium- and low-volatility states, respectively).

Table 2 also reports the monthly volatility autocorrelation in the three volatility states. Across major equity markets and factors, this autocorrelation tends to be much higher in high-volatility states than in other states, which suggests stronger volatility clustering in high-volatility states (for markets, on average 0.52 in the high-volatility state versus 0.14 and 0.23 in the medium- and low-volatility states; for momentum across regions, 0.60 in high-volatility states compared with 0.15 and 0.21 in medium- and low-volatility states). This finding implies stronger predictability of volatility in high-volatility statesenhancing the effectiveness of volatility targeting.

Overall, the results in Table 2 suggest that the gains from volatility targeting are likely to be highest during high-volatility states. This finding motivated us to construct volatility-targeting strategies that condition on (extreme) volatility states. The standard version of our conditional volatility-targeting strategy adjusts risk exposures in the extremes-that is, during high- and low-volatility states-and maintains an unscaled risk exposure otherwise. The weak correlation between realized volatility and future returns in the low-volatility state suggests that the lowest volatility is not necessarily associated with proportionally lower excess return, which provides scope for leverage. We capped the leverage in the low-volatility states to prevent an increase in overall risk and drawdowns (our initial strategy capped the maximum risk exposure at $200 \%$, but the finding is robust to different levels of leverage).

\section{Performance of Conditional Volatility Targeting}

In this section, we first discuss the performance and benefits of conditional volatility targeting in global equity markets and factors. Thereafter, we provide the robustness tests of our results.

\section{Performance of the Conditional}

Strategy. Table 3 shows the performance of the conditional portfolios relative to the original portfolios. As before, performance results that contradict the objectives of the strategy are in boldface. In Panel A, the first notable observation is that the conditional strategy significantly and consistently reduced the maximum drawdown across all equity markets (except the UK market), by an average of $6.6 \%$. It also consistently reduced the expected shortfall (99\%) of monthly returns in 9 out of the 10 markets, by an average of $1.3 \%$. This result is in contrast to the conventional strategy, which actually increased the maximum drawdown in the UK, Canadian, Australian, and Hong Kong markets (sometimes by a significant amount-e.g., 34.4\% in Hong Kong) and increased the expected shortfall in 8 out of 10 markets (a $2.3 \%$ increase, on average). To ensure that these results were not driven by reduced volatility, we also provide in Table 3 maximum drawdown and expected shortfall statistics with ex post scaling as in Moreira and Muir (2017) and confirm a significantly reduced maximum drawdown and expected shortfall.

The second observation is that the conditional strategy achieved a higher Sharpe ratio improvement than the conventional strategy $(0.07$ versus 0.04 , on average). In addition, the conditional strategy 


\section{Table 3. Performance of Conditional Volatility Targeting}

\begin{tabular}{|c|c|c|c|c|c|c|}
\hline \multicolumn{5}{|c|}{ Conditional Volatility-Targeting Portfolio } & \multicolumn{2}{|c|}{ Volatility Adjusted } \\
\hline $\begin{array}{l}\text { Sharpe } \\
\text { Ratio }\end{array}$ & $\begin{array}{l}\text { Maximum } \\
\text { Drawdown }\end{array}$ & $\begin{array}{l}\text { Expected } \\
\text { Shortfall }\end{array}$ & $\begin{array}{l}\text { Realiz } \\
\text { Volatil }\end{array}$ & & & $\begin{array}{l}\text { Exp } \\
\text { Shc }\end{array}$ \\
\hline Increase & Reduction & Reduction & Target & (times) & Reduction & Reduction \\
\hline
\end{tabular}

A. Global equity markets (1982-2019)

\begin{tabular}{|c|c|c|c|c|c|c|c|}
\hline US & $0.16^{*}$ & $-8.3 \%$ & $-1.7 \%$ & 0.97 & 1.6 & $-7.2 \%$ & $-1.3 \%$ \\
\hline Japan & 0.09 & -7.0 & -4.9 & 0.85 & 0.9 & -0.2 & -2.7 \\
\hline UK & 0.04 & 2.2 & -0.9 & 1.11 & 1.6 & -1.6 & -2.3 \\
\hline France & 0.03 & -5.1 & -0.3 & 0.92 & 1.4 & -1.9 & 1.1 \\
\hline Canada & 0.02 & -7.1 & -1.4 & 0.96 & 1.2 & -5.7 & -0.8 \\
\hline Germany & 0.07 & -10.4 & -2.4 & 0.86 & 1.1 & -4.3 & 0.6 \\
\hline Switzerland & 0.07 & -7.2 & -1.5 & 0.94 & 1.4 & -5.2 & -0.6 \\
\hline Australia & 0.04 & -7.8 & 2.7 & 1.12 & 1.5 & -12.1 & 0.5 \\
\hline Netherlands & 0.02 & -10.4 & -0.8 & 0.98 & 1.6 & -9.8 & -0.5 \\
\hline Hong Kong SAR & $0.14^{*}$ & -4.7 & -1.6 & 1.12 & 1.5 & -9.6 & -4.4 \\
\hline Market average & 0.07 & -6.6 & -1.3 & 0.98 & 1.4 & -5.8 & -1.0 \\
\hline \multicolumn{8}{|c|}{ B. US equity factors (1973-2019) } \\
\hline Size & -0.11 & $-0.6 \%$ & $-0.7 \%$ & 0.89 & 1.0 & $3.7 \%$ & $0.0 \%$ \\
\hline Value & -0.05 & 6.5 & -2.6 & 0.83 & 1.2 & 13.2 & -1.1 \\
\hline Profitability & -0.09 & -1.5 & -0.7 & 0.86 & 1.3 & 3.3 & 0.4 \\
\hline Investment & $-0.18^{* *}$ & 7.7 & -2.4 & 0.87 & 1.4 & 13.5 & -1.4 \\
\hline Momentum & $0.17^{* *}$ & -6.8 & -6.8 & 0.79 & 1.1 & 2.1 & -3.9 \\
\hline \multicolumn{8}{|c|}{ C. Global momentum factors (1995-2019) } \\
\hline Global & $0.19^{*}$ & $-21.0 \%$ & $-8.5 \%$ & 0.75 & 0.9 & $-12.5 \%$ & $-5.4 \%$ \\
\hline US & $0.26^{* *}$ & -18.3 & -12.1 & 0.75 & 1.2 & -9.3 & -8.5 \\
\hline Global ex US & $0.27^{* *}$ & -20.9 & -7.0 & 0.79 & 1.2 & -15.0 & -4.6 \\
\hline Europe & $0.27^{* *}$ & -21.2 & -9.0 & 0.74 & 1.1 & -12.9 & -5.6 \\
\hline Japan & 0.07 & -21.9 & -7.5 & 0.79 & 1.1 & -11.0 & -4.5 \\
\hline Pacific ex Japan & $0.30^{* *}$ & -17.0 & -12.1 & 0.95 & 1.6 & -15.2 & -11.1 \\
\hline $\begin{array}{c}\text { Momentum } \\
\text { average }\end{array}$ & 0.23 & -20.1 & -9.4 & 0.80 & 1.2 & -12.6 & -6.6 \\
\hline
\end{tabular}

Notes: Performance results that violate the objectives of the strategy are in boldface. The "Volatility Adjusted" statistics show the drawdown and shortfall reduction for a hypothetical conditional strategy with the same ex post realized volatility as the original portfolio. See Table 1 for descriptions of other column headings.

${ }^{*}$ Sharpe ratio increase significant at the $5 \%$ level.

${ }^{* *}$ Sharpe ratio increase significant at the $1 \%$ level.

improved Sharpe ratios in all markets, whereas the conventional strategy improved Sharpe ratios in 8 out of 10 markets. The caveat is that the Sharpe ratio improvement is statistically significant only for the US and Hong Kong markets. The performance consistency across all markets, however, reduces the concern that this outperformance is merely the result of chance. In addition, in practice, a single trading strategy on the aggregate market rarely improves Sharpe ratios with statistical significance. For this 
reason, most investors combine multiple strategies in their tactical asset allocation.

The third observation is that the annual turnover of the conditional strategy is much lower than that of the conventional strategy (e.g., 1.6 versus 2.4 for the US market and 1.4 versus 2.1, on average, across markets). Finally, the conditional strategy achieved a ratio of realized volatility to target volatility that is much closer to 1.0 (across-market average of 0.98 ), without a systematic bias of over- or undershooting.

In short, the conditional strategy consistently delivers its main promise of downside risk reduction, more significantly improves Sharpe ratios, and more accurately achieves the volatility target with materially lower turnover than the conventional strategy.

Panel B of Table 3 shows that the conditional volatility-targeting portfolio significantly improved performance for the US momentum factor but not for other US factors (consistent with the conventional strategy; see Table 1). It doubled the Sharpe ratio of US momentum, with an increase of 0.17 from 0.14 (statistically significant at the $1 \%$ level). This improvement is marginally greater than that of the conventional strategy $(+0.16)$ but comes at only half the turnover (1.1 versus 2).

Panel $\mathrm{C}$ of Table 3 presents the performance of the conditional strategy for momentum factors across regions for 1995-2019 (the US market is included for comparison). In this period, the momentum factors experienced high volatility and modest returns, with an average Sharpe ratio of only 0.12 across regions (see Table A1 in Appendix A). The conditional strategy more than doubled the Sharpe ratio for momentum, with an average increase of 0.23 . This increase is statistically significant at the $1 \%$ level for the US, global ex US, Europe, and Pacific ex Japan momentum factors and is significant at the $5 \%$ level for the global momentum factor. The conditional strategy significantly reduced maximum drawdowns, by an average of $20.1 \%$ (from $54.1 \%$ ), and reduced expected shortfall by an average of $9.4 \%$ (from $-20.8 \%$ ). After ex post rescaling, the maximum drawdown and expected shortfall reductions were still large (12.6\% and 6.6\%, respectively).

Compared with the conventional strategy (see Table A1 in Appendix A), the conditional strategy applied to the momentum factors achieved a Sharpe ratio increase that is higher in the US market $(0.26$ versus 0.16 ), lower in European markets ( 0.27 versus 0.37 ), and broadly similar in other regions-but with less regional variation. The conditional strategy incurred only half the turnover of the conventional strategy ( 1.2 versus 2.4 , on average), required materially lower leverage (maximum of 2.0 versus 5.5), and reduced expected shortfall more than the conventional strategy (9.4\% versus $7.2 \%)$. Moreover, for long-short portfolios, the conditional strategy is easier to implement in practice than the conventional strategy because taking leveraged positions in longshort equity factors is more challenging and costlier than in broad equity markets with liquid futures.

Many investors implement factor investing strategies by using long-only portfolios, in which the ability to underweight the undesired stocks is constrained by the stock's market-cap weight in the long-only equity benchmark. This constraint makes taking sizable leveraged positions on equity factors difficult. Therefore, we also considered an unlevered version of the conditional volatility strategy for momentum. This strategy reduced exposures only in highvolatility states. It maintained an unscaled exposure without taking leverage in all other states. ${ }^{17}$

To highlight the economic significance of conditional volatility targeting for the momentum factor,

Figure 1 allows a comparison of the annualized excess return and volatility of the (regular) conditional strategy and the unlevered conditional strategy with the original momentum factor. Both conditional strategies increased momentum's excess returns by an economically significant amount (from $1.2 \%$ to $3.1 \%$ and $4.5 \%$, respectively, in the US market; from $2.0 \%$ to $3.7 \%$ and $4.7 \%$, respectively, on average, across regions). The excess return improvement was achieved despite lower realized volatility. Hence, conditional volatility targeting can be used to enhance returns while reducing the risk budget for momentum factors.

Our key observations of stronger volatility clustering and strong correlation between realized volatility and future returns in high-volatility states apply not only to equity markets and the momentum factor but potentially also to other markets and factors. For instance, while equity markets and the momentum factor tend to crash in high-volatility states, treasury bonds and the profitability factor tend to generate positive returns during such flight-to-quality periods. Therefore, during high-volatility states, dynamic allocation from equities to treasury bonds and from momentum to the profitability factor has the potential to generate significant excess returns on top of the conditional volatility-targeting strategy. 
Figure 1. Economic Significance of Conditional Volatility Targeting for the Momentum Factor

\section{A. Annual Excess Return}

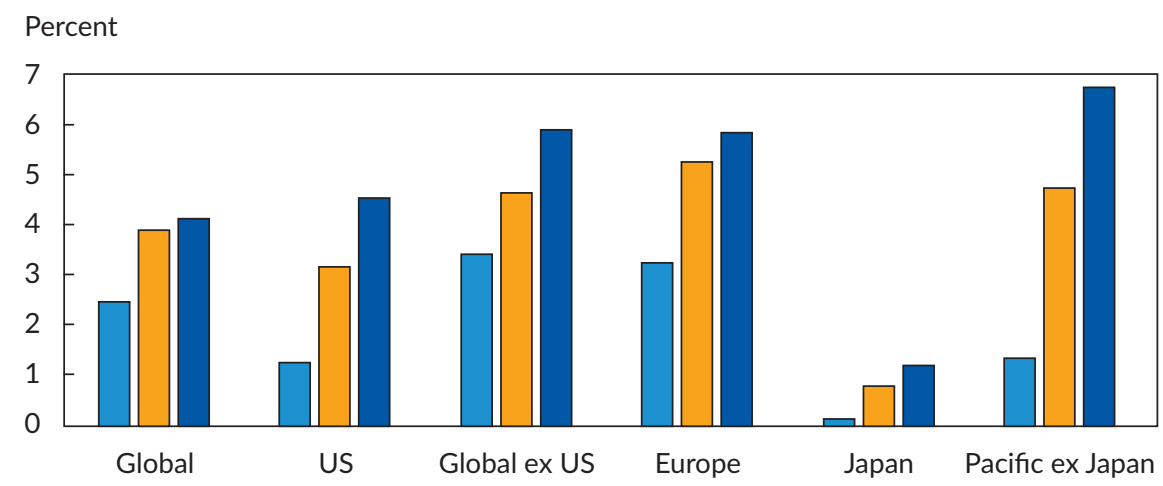

B. Realized Volatility

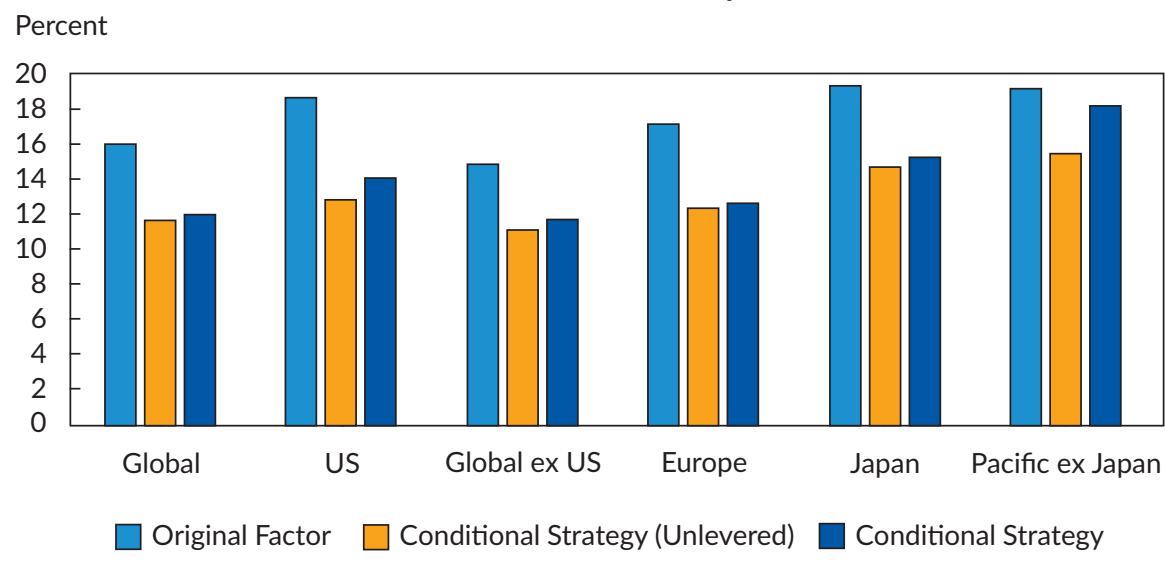

Notes: Performance is reported net of transaction costs. The sample period is $1995-2019$.
For instance, Figure A4 in Appendix A simulates a conditional strategy that reduces momentum exposure during high-volatility states and reallocates the capital to the profitability factor (instead of cash). This dynamic allocation to profitability generated additional excess return of about $30 \%$ during the technology bubble and about $15 \%$ during the global financial crisis. Similarly, we found that dynamic allocation to treasury bond futures during periods of high equity volatility generated additional returns. Such volatility management strategies with multiple asset classes or risk factors can be a promising direction for future research.

\section{Robustness Tests of Conditional}

Strategies. This section provides evidence that the performance of conditional volatility targeting with the momentum and market factors is persistent over long historical periods and robust to alternative approaches to identifying volatility states.
Our performance statistics in the sections "Motivation for Conditional Volatility Targeting" and "Performance of the Conditional Strategy" cover the entire sample period, which goes back to 1973 for US equity factors and 1982 for the 10 equity markets. Because investors generally have shorter horizons, we also analyzed the performance of conditional volatility targeting for the momentum and market factors over rolling 10 -year periods. ${ }^{18}$

As discussed in the section "Constructing VolatilityTargeting Portfolios," we estimated the volatility state of each month by using, for simplicity, realized volatility quintiles. We conducted a sensitivity test of this choice by using realized volatility quartiles instead of quintiles; we defined the top and bottom quartiles as, respectively, high-volatility and lowvolatility states. Table S2 in the Supplemental Online Appendix shows results that are very similar to the tests that used quintiles. 
In practice, investors may also estimate volatility states by using the ratio of realized volatility of the previous month to target volatility (i.e., defining high volatility as Realized volatility $>T_{h} \times$ Target volatility, where threshold $T_{h}$ is a constant). This method is not our preferred approach because this ratio has a heterogeneous distribution across various markets and equity factors, which implies that performance results would not be fully comparable across markets and factors. Nonetheless, as a robustness test, we analyzed the performance of conditional volatility targeting by using fixed thresholds of the ratio of realized volatility to target volatility. The medium realized volatility in a given month was only approximately 0.7 times the factor's long-term volatility because of the skewness in volatility distributions. For our robustness test, we used a ratio of 1.0 or 1.2 to define high-volatility states and a ratio of 0.5 or 0.6 to define low-volatility states.

Table 4 reports the performance improvement of the conditional strategy for the US market factor and momentum factor when we used three variations of volatility bands. The increase in Sharpe ratio and downside risk reduction are broadly consistent with those of the regular conditional strategy (Table 3) for both factors. In practice, given sufficient historical data on the volatility structure of a particular market or factor, investors can fine-tune the highvolatility/low-volatility bands to construct conditional strategies that meet particular risk and return requirements.

\section{Conclusions}

We analyzed volatility-targeting strategies in global equity markets and for factors. We found that conventional volatility-targeting strategies fail to consistently enhance risk-adjusted performance in global equity markets and can lead to markedly greater drawdowns than without volatility targeting. Conventional volatility targeting worked for momentum across regions but not for other factors, and it generated significant leverage and high turnover. We showed that these limitations of conventional volatility targeting stem mainly from its inability to condition on (extreme) volatility states.

Therefore, we constructed and analyzed volatilitytargeting strategies that conditioned on volatility states. We used equity index futures and investable equity factors that can be implemented by practitioners.

We showed that the benefits from volatility targeting are concentrated in high-volatility states. In these states, volatility clustering is stronger and the correlation between realized volatility and future return is significantly more negative than in low- or mediumvolatility states. Our conditional volatility-targeting strategy significantly improved Sharpe ratios for the momentum factor and, to a lesser degree, the market factor across regions. The conditional strategy also consistently reduced maximum drawdowns and tail risks and did so with much lower turnover and leverage than the conventional strategy.

\section{Table 4. Performance of Conditional Volatility Targeting with Various Ratios of Realized} Volatility to Target Volatility

\begin{tabular}{cccccccc} 
& $\begin{array}{c}\text { High- } \\
\text { Volatility } \\
\text { Band }\end{array}$ & $\begin{array}{c}\text { Low-Volatility } \\
\text { Band }\end{array}$ & $\begin{array}{c}\text { Sharpe } \\
\text { Ratio } \\
\text { Increase }\end{array}$ & $\begin{array}{c}\text { Maximum } \\
\text { Drawdown } \\
\text { Reduction }\end{array}$ & $\begin{array}{c}\text { Expected } \\
\text { Shortfall } \\
\text { Reduction }\end{array}$ & $\begin{array}{c}\text { Realized } \\
\text { Volatility/ } \\
\text { Target }\end{array}$ & $\begin{array}{c}\text { Annual } \\
\text { Turnover }\end{array}$ \\
\hline \multirow{3}{*}{ US market } & $>1.0 \times$ Target & $<0.5 \times$ Target & $0.15^{*}$ & $-8.5 \%$ & $-2.4 \%$ & 0.93 & 1.36 \\
& $>1.2 \times$ Target & $<0.5 \times$ Target & $0.15^{*}$ & -8.7 & -2.3 & 0.95 & 1.33 \\
& $>1.0 \times$ Target & $<0.6 \times$ Target & $0.17^{*}$ & -8.5 & -1.7 & 1.00 & 1.75 \\
\multirow{3}{*}{ US momentum } & $>1.0 \times$ Target & $<0.5 \times$ Target & $0.15^{* *}$ & $-5.9 \%$ & $-6.9 \%$ & 0.79 & 1.23 \\
& $>1.2 \times$ Target & $<0.5 \times$ Target & $0.15^{* *}$ & -6.1 & -6.7 & 0.81 & 1.19 \\
& $>1.0 \times$ Target & $<0.6 \times$ Target & $0.17^{* *}$ & -11.1 & -6.2 & 0.84 & 1.52 \\
\hline
\end{tabular}

Notes: See the notes to Table 1. Performance is reported relative to the original portfolio and net of transaction costs.

*Sharpe ratio increase significant at the $5 \%$ level.

** Sharpe ratio increase significant at the $1 \%$ level. 
The conditional volatility-targeting strategy has several practical applications in factor investing and tactical asset allocation. The momentum factors have generated relatively modest returns with significant volatility and large drawdowns in recent decades.

Using the conditional strategy for momentum factors could generate economically large improvements in returns with materially lower downside risks. Similarly, against the current backdrop of low expected returns and high uncertainties in global equity markets, the conditional strategy can be used to enhance the riskadjusted performance of broad equity markets.
Conditional volatility management has broader applications beyond our main analysis. For instance, it can be applied to tactical allocation between risky assets (equities, the momentum factor) and flight-to-quality assets (treasuries, the profitability factor), which offers to further improve performance, particularly during high-volatility states. Finally, we believe the performance of conditional volatility management can be further enhanced through advanced methods of volatility-state forecasting.

\section{Appendix A. Additional Performance Analysis}

Figure A1. Volatility Clustering and Negative Correlation between Equity Returns and Volatility

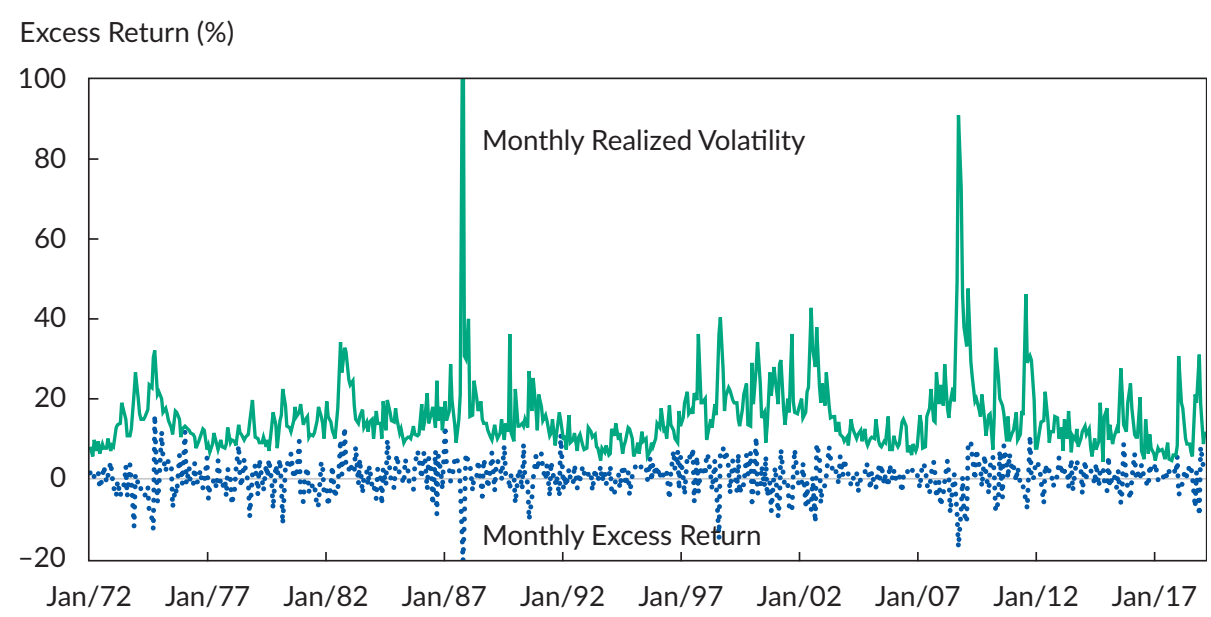

Figure A2. Ratio of Realized Monthly Volatility to Target Volatility for Conventional VolatilityTargeting Strategy on S\&P 500

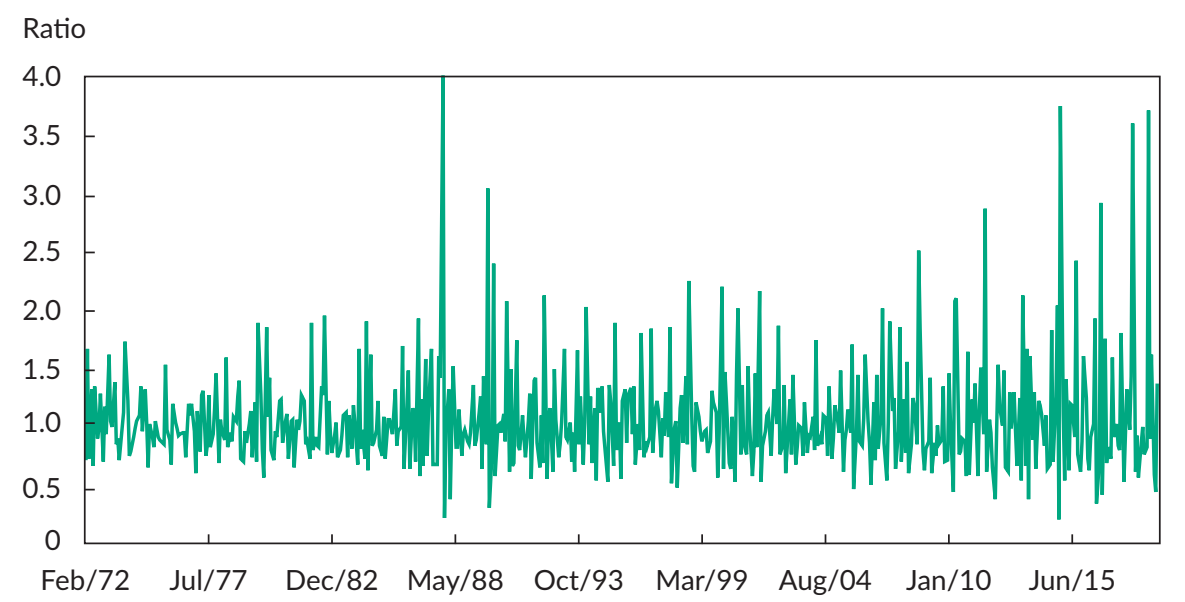

Note: This figure shows the time series of the ratio of monthly realized volatility of the conventional volatility-targeting strategy to the target volatility (unconditional volatility of the S\&P 500). 


\section{Table A1. Performance of Conventional Volatility Targeting on Global Momentum Factors}

\begin{tabular}{|c|c|c|c|c|c|c|c|}
\hline \multicolumn{3}{|c|}{ Original Portfolio } & \multicolumn{5}{|c|}{ Conventional Volatility-Targeting Portfolio } \\
\hline $\begin{array}{l}\text { har } \\
\text { Rati }\end{array}$ & $\begin{array}{l}\text { Maximum } \\
\text { Drawdown }\end{array}$ & Shortfall & $\begin{array}{l}\text { Sharpe } \\
\text { Ratio } \\
\text { Increase }\end{array}$ & & $\begin{array}{l}\text { Expected } \\
\text { Shortfall } \\
\text { Reduction }\end{array}$ & $\begin{array}{l}\text { Realized } \\
\text { Volatility/ } \\
\text { Target }\end{array}$ & $\begin{array}{c}\text { Annual } \\
\text { Turnover } \\
\text { (times) }\end{array}$ \\
\hline
\end{tabular}

\begin{tabular}{lllllllll}
\hline Global momentum factors (1995-2019) & & & & & & \\
Global & 0.15 & $52.3 \%$ & $-18.2 \%$ & $0.17^{*}$ & $-21.0 \%$ & $-7.4 \%$ & 0.97 & 2.5 \\
US & 0.07 & 51.3 & -23.1 & 0.16 & -19.3 & -10.4 & 0.84 & 2.0 \\
Global ex US & 0.23 & 47.0 & -16.0 & $0.28^{* *}$ & -21.9 & -6.0 & 1.01 & 2.4 \\
Europe & 0.19 & 49.3 & -18.8 & $0.37^{* *}$ & -20.9 & -7.7 & 0.97 & 2.4 \\
Japan & 0.01 & 62.2 & -19.0 & 0.04 & -20.4 & -3.7 & 1.04 & 2.6 \\
Pacific ex Japan & 0.07 & 62.3 & -29.9 & $0.34^{* *}$ & -15.8 & -7.9 & 1.16 & 2.4 \\
\multicolumn{1}{l}{ Average } & 0.12 & 54.1 & -20.8 & 0.23 & -19.9 & -7.2 & 1.00 & 2.4
\end{tabular}

Notes: See the notes to Table 1. The table is complementary to Table 1 and shows the performance of the conventional strategy (net of transaction costs) relative to that of the original portfolio.

*Sharpe ratio increase significant at the $5 \%$ level.

** Sharpe ratio increase significant at the $1 \%$ level.

Figure A3. Historical

Return and Volatility of US Profitability and Momentum Factors

\section{A. US Profitability Factor}

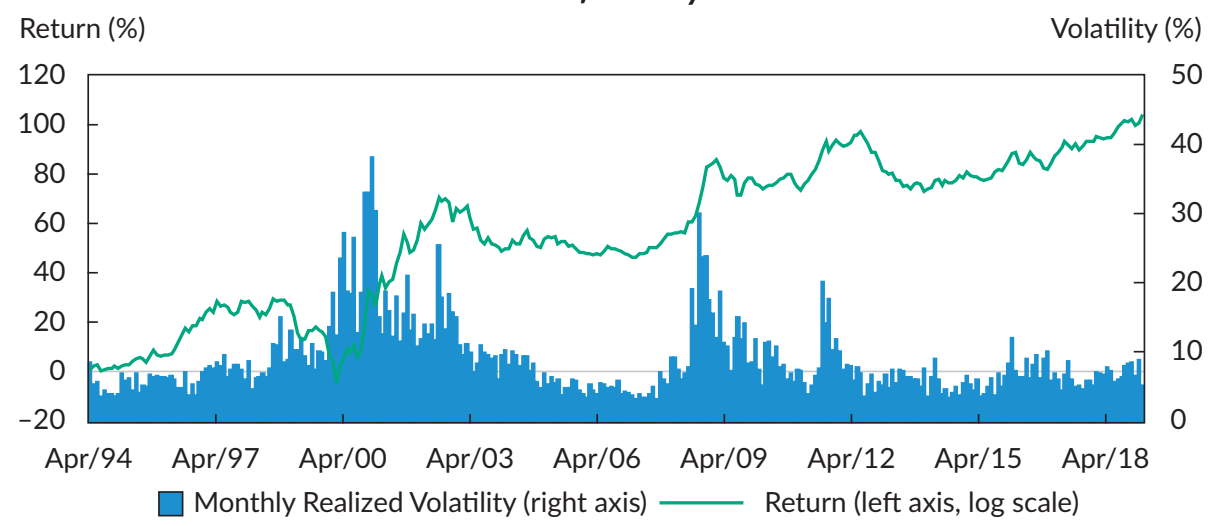

\section{B. US Momentum Factor}

Return (\%)

Volatility (\%)

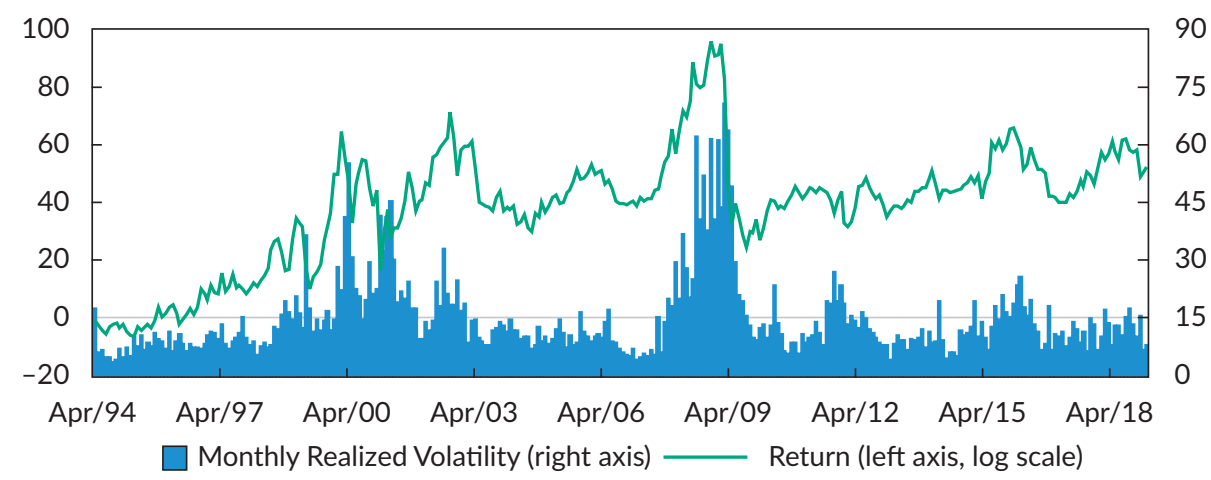




\begin{tabular}{|c|c|c|c|c|c|c|c|c|}
\hline & \multicolumn{3}{|c|}{ Original Portfolio } & \multicolumn{5}{|c|}{ Conditional Volatility-Targeting Portfolio } \\
\hline & $\begin{array}{l}\text { Sharpe } \\
\text { Ratio }\end{array}$ & $\begin{array}{l}\text { Maximum } \\
\text { Drawdown }\end{array}$ & $\begin{array}{l}\text { Expected } \\
\text { Shortfall }\end{array}$ & $\begin{array}{l}\text { Sharpe } \\
\text { Ratio } \\
\text { Increase }\end{array}$ & $\begin{array}{l}\text { Maximum } \\
\text { Drawdown } \\
\text { Reduction }\end{array}$ & $\begin{array}{l}\text { Expected } \\
\text { Shortfall } \\
\text { Reduction }\end{array}$ & $\begin{array}{c}\text { Realized } \\
\text { Volatility/ } \\
\text { Target }\end{array}$ & $\begin{array}{l}\text { Annual } \\
\text { Turnover } \\
\text { (times) }\end{array}$ \\
\hline \multicolumn{9}{|c|}{ Global momentum factors (1995-2019) } \\
\hline Global & 0.15 & $52.3 \%$ & $-18.2 \%$ & $0.18^{*}$ & $-21.0 \%$ & $-8.5 \%$ & 0.73 & 0.40 \\
\hline US & 0.07 & 51.3 & -23.1 & $0.18^{*}$ & -18.3 & -12.1 & 0.68 & 0.45 \\
\hline Global ex US & 0.23 & 47.0 & -16.0 & $0.18^{*}$ & -20.9 & -7.0 & 0.75 & 0.40 \\
\hline Europe & 0.19 & 49.3 & -18.8 & $0.24^{* *}$ & -21.2 & -9.0 & 0.72 & 0.44 \\
\hline Japan & 0.01 & 62.2 & -19.0 & 0.05 & -17.2 & -7.6 & 0.76 & 0.40 \\
\hline Pacific ex Japan & 0.07 & 62.3 & -29.9 & $0.24^{* *}$ & -21.0 & -12.1 & 0.81 & 0.26 \\
\hline Average & 0.12 & 54.1 & -20.8 & 0.18 & -19.9 & -9.4 & 0.74 & 0.39 \\
\hline
\end{tabular}

Notes: See the notes to Table 1. The table shows the performance of the unlevered conventional strategy that adjusts risks only during high-volatility states, net of transaction costs.

${ }^{*}$ Sharpe ratio increase significant at the $5 \%$ level.

${ }^{* *}$ Sharpe ratio increase significant at the $1 \%$ level.

Figure A4. Additional Excess Return (\%)

Volatility (\%)

Excess Return from

Tactical Allocation to

Profitability Factor

during High-Volatility

States

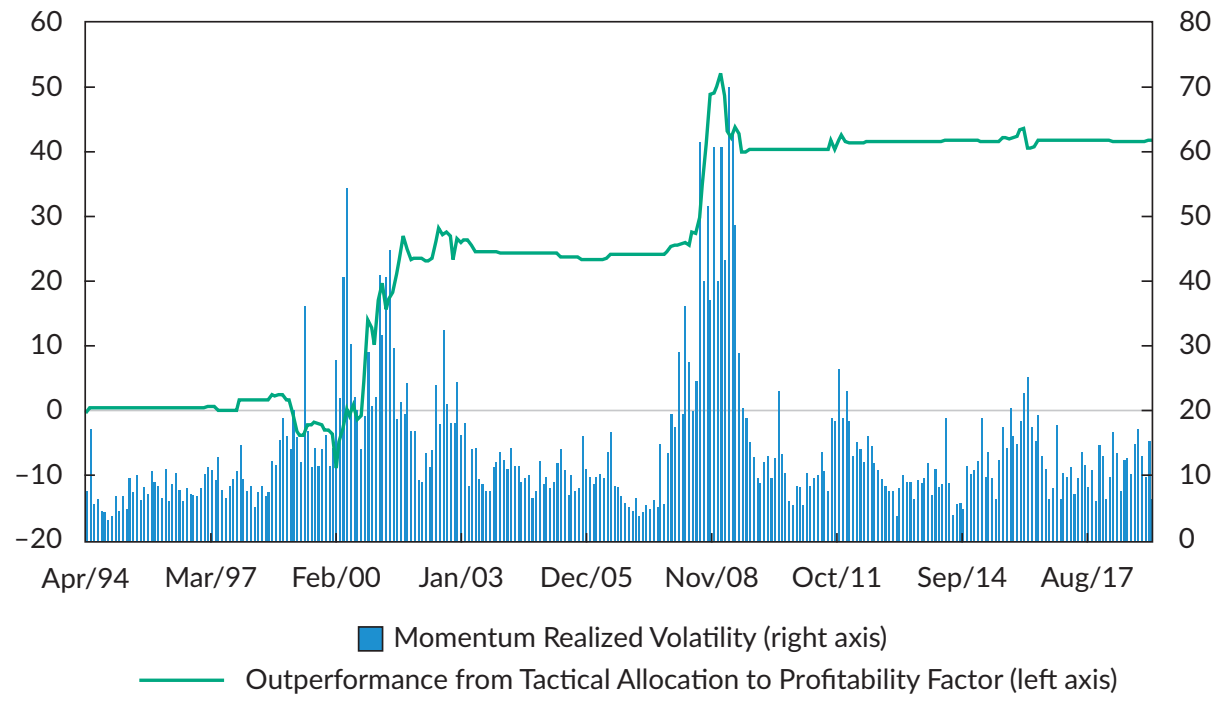

Notes: This figure simulates the performance of a conditional strategy that reduces momentum exposure during high-volatility states and reallocates the capital to the profitability factor (instead of cash). It shows the additional return from the tactical allocation to the profitability factor, in excess of the performance of the regular conditional strategy. 
Figure A5. Sharpe Ratio over Rolling 10-Year Periods for US Momentum and US Market

\section{A. US Momentum: Sharpe Ratio over Rolling 10-Year Periods} Sharpe Ratio

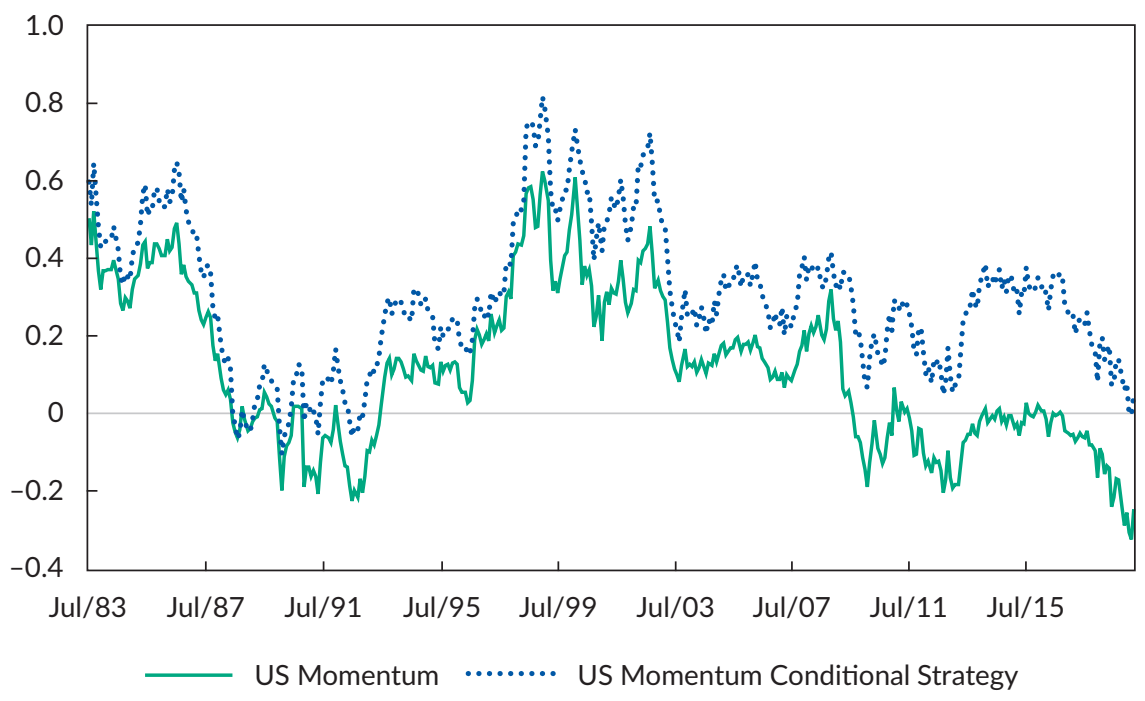

\section{B. US Market: Sharpe Ratio over Rolling 10-Year Periods}

Sharpe Ratio

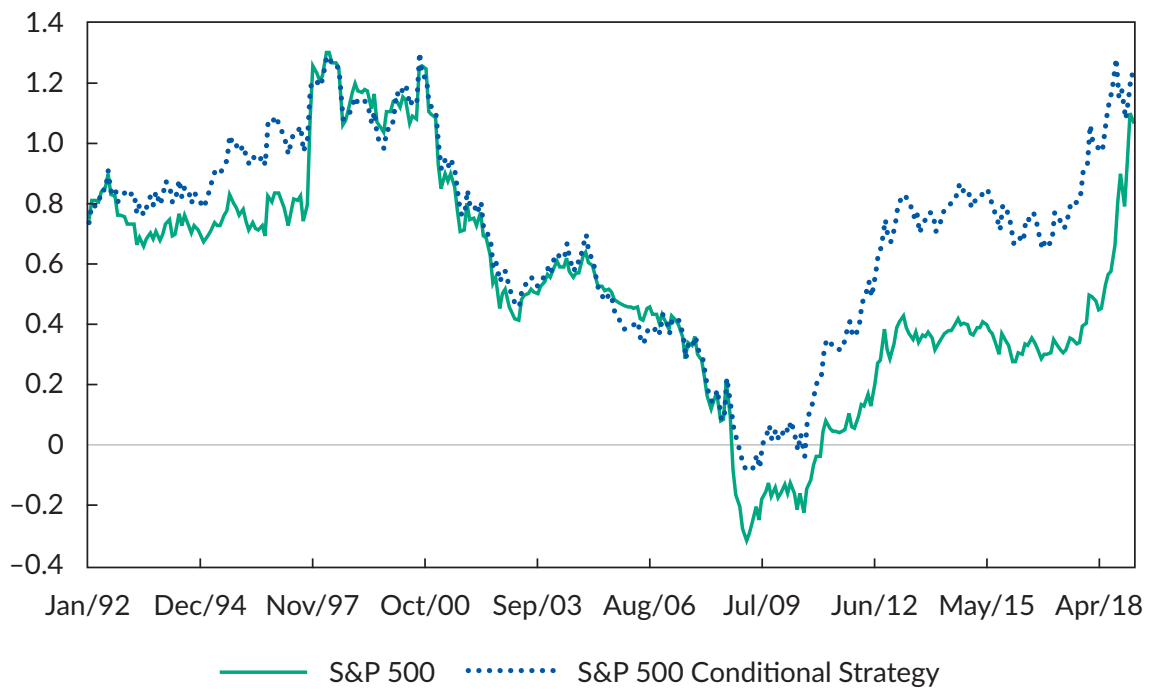

\section{Editor's Note}

This article was externally reviewed using our double-blind peer-review process. When the article was accepted for publication, the authors thanked the reviewers in their acknowledgments. Lisa Goldberg and one anonymous reviewer were the reviewers for this article.

Submitted 6 March 2020

Accepted 23 June 2020 by Stephen J. Brown

\section{Notes}

1. We ranked equity markets by free-float market capitalization as of March 2019, and we selected the 10 largest markets for which we had return data for the whole sample period-that is, since 1972 .

2. This approach did not affect the accuracy of our results because of the high correlation (0.99) between an equity index and its futures. Moskowitz, Ooi, and Pedersen (2012) also combined futures data with equity index data.

3. The French website is https://mba.tuck.dartmouth.edu/ pages/faculty/ken.french/data_library.html. 
4. For completeness, we show in the Supplemental Online Appendix (Table S1) that empirical results when the original factors were used are consistent with our findings.

5. As illustrated by Anderson, Bianchi, and Goldberg (2012), levering a strategy by a constant leaves the Sharpe ratio in tact only if the borrowing rate equals the risk-free rate. This assumption applies to volatility-targeting strategies with equity factors that require borrowing/leverage.

6. We include in Table A2 in Appendix A an unlevered version of the conditional strategy that adjusted risks only in high-volatility states. This approach can be useful to investors who are constrained not to use leverage because of investment guidelines or cost considerations.

7. For equity factors in international markets, our sample period began in November 1990. To avoid a further shortening of our sample period, we required at least five years of historical returns to calculate long-term volatilities for those markets.

8. Using monthly realized volatility produced somewhat better performance than using exponentially weighted volatility or GARCH for the conventional and the conditional strategies. The reason is that realized volatility responds more quickly to market turmoil.

9. Their methodology accounted for all trading costs, including commissions, bid-ask spreads, and market impact.

10. Figure $A 1$ in Appendix A illustrates the association of major market crashes-such as Black Monday in 1987, the bursting of the tech bubble, the 2008 global financial crisis, and the European debt crisis-with large volatility spikes and strong volatility clustering.

11. In contrast, Campbell and Hentschel (1992) developed a model of volatility feedback effects in which an increase in equity volatility raises the required return on equity and thus brings about a decline in equity price.

12. Hallerbach (2012) and Dachraoui (2018) also discussed the conditions for volatility targeting to improve risk-adjusted performance.

13. Expected shortfall is preferable to value at risk (VAR) because expected shortfall is the mean of losses beyond the percentile whereas VAR disregards losses beyond the percentile. See Acerbi and Tasche (2002).

14. See also Figure A2 in Appendix A.

15. Daniel and Moskowitz (2016), studying momentum crashes, designed a dynamic strategy based on forecasts of momentum's mean and variance to increase the Sharpe ratio of a static momentum strategy.

16. See Table A1 in Appendix A.

17. Table A2 in Appendix A shows that this unlevered conditional strategy still doubled the Sharpe ratio, increasing it by an average of 0.18 , with statistical significance in all regions except Japan. It required only a fraction of the turnover of the conventional strategy $(0.4$ versus 2.4 , on average) and achieved more significant downside risk reduction.

18. Figure A5 in Appendix A shows that the increase in Sharpe ratios is highly persistent for the US momentum factor (Panel A) and the US market (Panel B, although it is a bit weaker) over the rolling periods. Contrary to rebuttals that volatility targeting "buys high and sells low," these results indicate that the strategy's outperformance tends to increase during market crashes because it quickly reduces risks when a crisis hits and increases risk when the market starts to recover.

\section{References}

Acerbi, C., and D. Tasche. 2002. "On the Coherence of Expected Shortfall." Journal of Banking \& Finance 26 (7): 1487-503.

Anderson, R. M., S. W. Bianchi, and L. R. Goldberg. 2012. "Will My Risk Parity Strategy Outperform?" Financial Analysts Journal 68 (6): 75-93.

Ang, A., and G. Bekaert. 2004. "How Regimes Affect Asset Allocation." Financial Analysts Journal 60 (2): 86-99.

Ang, A., W. N. Goetzmann, and S. M. Schaefer. 2009. "Evaluation of Active Management of the Norwegian Government Pension Fund-Global." Working paper (14 December).

Black, F. 1976. "Studies of Stock Price Volatility Changes." Proceedings of the 1976 Business and Economic Statistics Section, American Statistical Association: 177-81.

Campbell, J. Y., and L. Hentschel. 1992. "No News Is Good News: An Asymmetric Model of Changing Volatility in Stock Returns." Journal of Financial Economics 31 (3): 281-318.

Carhart, M. M. 1997. "On Persistence in Mutual Fund Performance." Journal of Finance 52 (1): 57-82.

Cederburg, S., M. S. O'Doherty, F. Wang, and X. Yan. Forthcoming. "On the Performance of Volatility-Managed Portfolios." Journal of Financial Economics.

Chen, Y., and B. Liang. 2007. "Do Market Timing Hedge Funds Time the Market?" Journal of Financial and Quantitative Analysis 42 (4): 827-56.

CME Group. 2016. "A Cost Comparison of Futures and ETFs." CME Group Big Picture Papers.

Dachraoui, K. 2018. "On the Optimality of Target Volatility Strategies." Journal of Portfolio Management 44 (5):58-67.

Daniel, K., and T. J. Moskowitz. 2016. "Momentum Crashes." Journal of Financial Economics 122 (2): 221-47.

Engle, R. F. 1982. "Autoregressive Conditional Heteroskedasticity with Estimates of the Variance of U.K. Inflation." Econometrica 50 (4): 987-1007. 
Fama, E. F., and K. R. French. 2015. "A Five-Factor Asset Pricing Model." Journal of Financial Economics 116 (1): 1-22.

Frazzini, A., R. Israel, and T. J. Moskowitz. 2015. "Trading Costs of Asset Pricing Anomalies." Working paper, AQR Capital Management.

Hallerbach, W. G. 2012. "A Proof of the Optimality of Volatility Weighting over Time." Journal of Investment Strategies 1 (4): 87-99.

Harvey, C. R., E. Hoyle, R. Korgaonkar, S. Rattray, M. Sargaison, and O. Van Hemert. 2018. "The Impact of Volatility Targeting." Journal of Portfolio Management 45 (1): 14-33.

Henkel, S. J., J. S. Martin, and F. Nardari. 2011. "Time-Varying Short-Horizon Predictability." Journal of Financial Economics 99 (3): 560-80.

Kotecha, C., C. Couasnon, and J. Galazidis. 2016. "A Cost Comparison of Futures, ETFs, and Swaps on Equity Indices." Bank of America Merrill Lynch Delta-One Cost Monitor.
Kritzman, M. P., S. Page, and D. Turkington. 2012. "Regime Shifts: Implications for Dynamic Strategies." Financial Analysts Journal 68 (3): 22-39.

Liu, F., X. Tang, and G. Zhou. 2019. "Volatility-Managed Portfolio: Does It Really Work?" Journal of Portfolio Management 46 (1): 38-51.

Lo, A. W. 2002. "The Statistics of Sharpe Ratios." Financial Analysts Journal 58 (4): 36-52.

Moreira, A., and T. Muir. 2017. "Volatility-Managed Portfolios." Journal of Finance 72 (4): 1611-44.

Moskowitz, T. J., Y. H. Ooi, and L. H. Pedersen. 2012. "TimeSeries Momentum." Journal of Financial Economics 104 (2): 228-50. 